\section{§19. Analysis of Carbon Deposition on the First Wall of LHD by Monte Carlo Simulation}

Kawamura, G., Tomita, Y., Kobayashi, M., Tokitani, M., Masuzaki, S.,

Kirschner, A. (Forschungszentrum Jülich $\mathrm{GmbH}$ )

Deposition of impurities on surfaces of plasma confinement devices is one of essential issues for long-time discharges and reduction of tritium inventory in present devices and also future fusion devices. The main components of the deposited impurities on the LHD first wall are carbon and iron during the main and glow discharges, respectively ${ }^{1,2,3)}$. Although the deposition itself does not cause a problem to the plasma directly, a long-term accumulation of impurities on the device surfaces can harm the plasma if the deposited films peel off into the plasma. In order to understand the characteristics of the impurity deposition, estimate and control impurity behaviors, simulation studies are necessary along with experimental investigations.

We employed the ERO code ${ }^{4)}$ to reveal how impurity carbon is transported and where it deposits. The Monte Carlo code ERO traces impurity atoms and molecules in simulation space with a number of atomic processes such as ionization and dissociation. The electromagnetic force and the collisional forces due to the background hydrogen plasma in the simulation box are involved in the calculations. We use the 2D model given in our previous report and curried out the Monte Carlo simulation. The ERO code traces impurity particles of various charge state and chemical species such as $\mathrm{C}, \mathrm{C}^{+}$, $\mathrm{C}^{2+}, \mathrm{C}^{3+}, \mathrm{C}^{4+}, \mathrm{CH}, \mathrm{CH}_{2}^{+}$and so on. We note that $\mathrm{C}^{5+}$ and $\mathrm{C}^{6+}$ are not generated because of insufficient electron temperature for further ionization. Since carbon is easily ionized by electrons, the neutral carbon is quite low density in the divertor leg. The perpendicular width of the leg is, however, relatively short and thus a certain amount of carbon atoms can penetrate the plasma without ionization and reach the first wall directly.

The deposition profiles on the wall are shown in Fig. 1(a). The solid and dashed lines represent the number of carbon atoms per area of each surface mesh coming from the adjacent and opposite divertors, respectively. The divertor plate region, $-0.33[\mathrm{~m}]<x<[\mathrm{m}]-0.27$, is omitted in the figure because the plasma-wetted surface has large erosion and there are no net depositions. The deposition caused by the opposite divertor is much less than that caused by the adjacent one on the first wall, while opposite divertor makes a major contribution in the outer region, i.e. $x<-0.33[\mathrm{~m}]$. The difference comes from the geometry of the LHD divertor. The outer region is located behind the divertor and thus the impurities come only from the opposite divertor. If the amount of deposition is determined only by geometrical

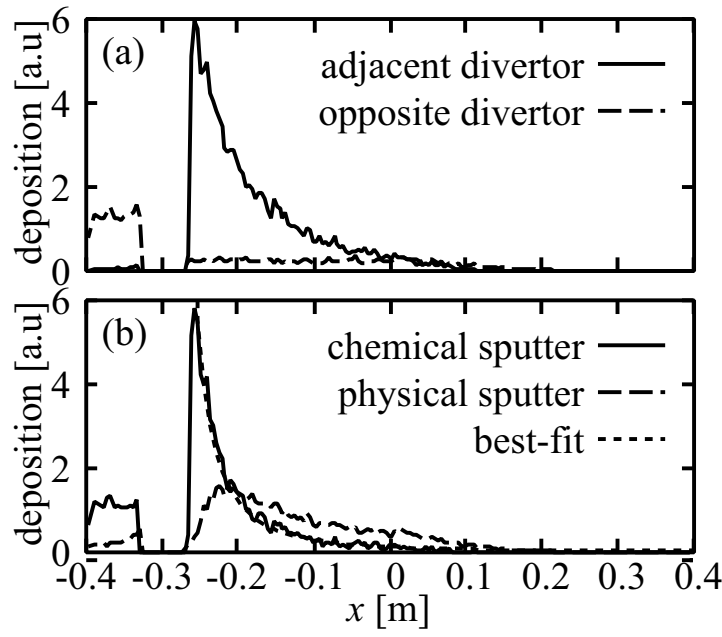

Fig. 1: Deposition profiles of carbon; (a) comparison of source positions, (b) comparison of chemical/physical sputtering. The best-fit curve is given by $0.012(x+0.3)^{-2}$ in the range of $x>-0.33[\mathrm{~m}]$.

reason, the deposition on the first wall due to the opposite divertor must be at the same level as that on the outer region, but that is not the case. The reason is the presence of another divertor leg between the first wall and the opposite divertor plate. Since ionization in the plasma prevents the impurity particles from reaching the first wall, the deposition is significantly reduced.

The composition of the deposition layer is strongly affected by the impurity species. Figure 1(b) shows the amount of carbon resulting from chemical and physical sputtering. Their profiles differ from each other especially near the divertor plate. The chemically sputtered carbon has a sharp peak at $x \simeq-0.25[\mathrm{~m}]$, while physical one does not have a peak and the decay length to the $x$ direction is much longer. The dotted line in the figure is an inverse-square curve and coincides with the deposition profile of the chemically sputtered carbon. That implies the incident particles had isotropic velocities when they were generated near the strike point. At the same time the fact that charged particles cannot leave the plasma for the first wall indicates that the deposition is caused by neutral radicals and atoms, i.e. $\mathrm{CH}_{3}, \mathrm{CH}_{2}, \mathrm{CH}$ and $\mathrm{C}$. That is consistent with the isotropic velocity distribution because the generation of the neutral particles requires atomic processes such as ionization and dissociative recombination, which lead to isotropic particle velocities.

1) A. Sagara, S. Masuzaki, T. Morisaki et al., J. Nucl. Mater. 313-316, 1 (2003)

2) T. Hino, Y. Nobuta,Y. Yamauchi, et al., J. Nucl. Mater. 313-316, 167 (2003)

3) Y. Nobuta, Y. Yamauchi, Y. Hirohata et al., J. Nucl. Mater. 329-333, 800 (2004)

4) A. Kirschner, V. Philipps, J. Winter and U. K. Kögler, Nucl. Fusion 40 (2000) 989. 